\title{
Performance Comparison between OQAM and $N$-continuous OFDM
}

\author{
Guobing Cheng, Huilei Li, Shaoqian Li, Lisha Gong, Binhong Dong, Peng Wei \\ National Key Laboratory of Science and Technology on Communications, \\ University of Electronic Science and Technology of China, Chengdu, China \\ Email: guobingcheng12@163.com
}

Received August, 2013

\begin{abstract}
Orthogonal frequency division multiplex/offset QAM (OFDM/OQAM) and $N$-continuous OFDM are both improved multi-carrier modulation (MCM) techniques for the transmission of signals over multipath fading channels. In this paper, we aim to compare these two techniques with respect to out-of-band radiation, bit error ratio (BER) and calculation complexity. Analysis and simulation results show that, compared to the $N$-continuous OFDM, the OFDM/OQAM has lower out-of-band radiation, calculation consumption and similar BER performance.
\end{abstract}

Keywords: OFDM/OQAM; N-continuous OFDM; Out-of-band Radiation

\section{Introduction}

Orthogonal frequency division multiplexing (OFDM) is an efficient scheme and it has been some practical applications. However, the traditional OFDM has some intrinsic drawbacks that the discontinuous phase between adjacent symbols leads to high out-of-band radiations and its robustness to multi-path propagation effect is acquired by the insertion of a cyclic prefix (CP). To alleviate these drawbacks, another MCM schemes such as $\mathrm{N}$-continuous OFDM and OFDM/offset QAM (OFDM/OQAM) are proposed in $[1,2]$ respectively. Compared to traditional CP-OFDM, OFDM/OQAM may provide a higher useful bit rate since it operates without $\mathrm{CP}$ and $\mathrm{N}$-continuous OFDM has less out-of-band radiation since it has several continuous order of derivatives at the edge of adjacent OFDM symbols.

For OFDM/OQAM system, pulse shapes with good time-frequency localization (TFL) can be introduced. Among these, a pulse shape named extended Gaussian functions (EGF) is widely used and its TFL can be adjusted by the time and frequency real parameters [3]. Through selecting a suitable parameter, an OFDM/ OQAM signal with very low out-of-band radiation can be acquired [4]. On the other hand, in the traditional OFDM system, each OFDM symbol is independently and the transmitted signals are not continuous, leading to high out-of-band radiation. In order to overcome the discontinuities between consecutive OFDM symbols, Ncontinuous OFDM scheme is proposed at the price of increasing calculation complexity and/or system per- formance. Therefore, we have to comprise between these aspects for practical application.

In this paper, we compare the performance of OFDM/ OQAM and N-continuous OFDM. Firstly, the system models of them are given in section II. And then we analyze the out-of-band radiation, bit error ratio (BER) and calculation complexity of these two schemes in section III. The simulation results are shown in section IV. Brief conclusion is given in section V. The analysis and simulation results show that, compared to the $\mathrm{N}$-continuous OFDM, the OFDM/OQAM system has similar system performance, lower out-of-band radiation and less calculation consumption.

\section{System Model}

\subsection{System Model of OFDM/OQAM System}

The baseband version of a continuous-time OFDM/ OQAM transmitting signal can be written as [2]

$$
s(t)=\sum_{n=-\infty}^{+\infty} \sum_{m=0}^{M-1} a_{m, n} e^{j \phi_{m, n}} e^{j 2 \pi m v_{0} t} g\left(t-n \tau_{0}\right),
$$

with $M$ an even number of sub-carriers, $a_{m, n}$ the realvalued symbol conveyed by the sub-carrier of index $m$ during the symbol time of index $n v_{0}$ the subcarrier spacing and $\tau_{0}$ the time offset between the adjacent real part and imaginary part of an OFDM/OQAM symbol. $v_{0}=1 / T_{0}=1 / 2 \tau_{0}$, with $T_{0}$ the duration of the complexvalued symbols. $\phi_{m, n}$ is an additional phase term given by 


$$
\phi_{m, n}=\phi_{0}+\frac{\pi}{2}(m+n)(\bmod \pi),
$$

where $\phi_{0}$ can be arbitrarily chosen. $g(t)$ is the pulse shape that can be different from the rectangle shape of conditional OFDM system. The EGF shape is often used in OFDM/OQAM system and its out-of-band characteristic can be adjusted by the Gaussian parameter $\alpha$. In the following, the adaption and compromise between BER performance and out-of-band radiation are discussed.

For a distortion-free channel, perfect reconstruction of real symbols is obtained owing to the following real orthogonal condition

$$
\mathfrak{R}\left\{\left\langle g_{m, n} \mid g_{p, q}\right\rangle\right\}=\mathfrak{R}\left\{\int g_{m, n}(t) g_{p, q}^{*}(t)\right\}=\delta_{m, p} \delta_{n, q}
$$

where $\mathfrak{R}\{\bullet\}$ is the taking real part operator. $\delta_{m, p}=1$ if $m=p$ and $\delta_{m, p}=0$ if $m \neq p$.

\subsection{System model of $\mathrm{N}$-continuous OFDM System}

The baseband equivalent OFDM symbol can be given as

$$
s(t)=\sum_{i=-0}^{+\infty} s_{i}\left(t-i\left(T_{s}+T_{g}\right)\right)
$$

where $T_{s}$ is the OFDM symbol duration and $T_{g}$ is the CP length. In order to make the transmitted signal $s(t)$ and its first $\mathrm{N}$ derivatives continuous, the following equation should be satisfied that

$$
\left.\frac{d^{n}}{d t^{n}} S_{i}(t)\right|_{t=-T_{g}}=\left.\frac{d^{n}}{d t^{n}} S_{i-1}(t)\right|_{t=T_{g}},
$$

for all $i \geq 1$ and $n=0,1, \ldots, \mathrm{N}[1,5]$.

The realization of $\mathrm{N}$-continuous OFDM symbol is to pre-coder the set of information symbols $\left\{d_{k, i}\right\}$ (taken from some complex-valued symbol constellation $\mathbb{C}$ ) in each OFDM symbol such that $s(t)$ becomes $\mathrm{N}$-continuous. Concretely, the OFDM symbol $s_{i}(t)$ can be expressed as [1]

$$
S_{i}(t)=\sum_{k \in \Omega} \bar{d}_{k, i} e^{j 2 \pi \frac{k}{T_{s}} t}-T_{g} \leq t \leq T_{s},
$$

where $\Omega=\left\{k_{0}, k_{1}, \ldots, k_{K-1}\right\}$ and the complex numbers $\bar{d}_{k, i}$ are the result of pre-coding information symbols $d_{k, i}$. For OFDM symbols, the characterization (5) becomes

$$
\sum_{k \in \Omega} k^{n} e^{j \phi k} \bar{d}_{k, i}=\sum_{k \in \Omega} k^{n} \bar{d}_{k, i-1},
$$

where $\phi=-2 \pi T_{g} / T_{s}$. An equivalent form of vector is that

$$
\mathbf{A} \Phi \overline{\mathbf{d}}_{\mathbf{i}}=\mathbf{A} \overline{\mathbf{d}}_{\mathbf{i}-1}
$$

where

$$
\begin{gathered}
\overline{\mathbf{d}}_{\mathbf{i}}=\left(\bar{d}_{k_{0}, i}, \bar{d}_{k_{1}, i}, \ldots, \bar{d}_{k_{K-1}, i}\right)^{T}, \\
\Phi=\operatorname{diag}\left(e^{j \phi k_{0}}, e^{j \phi k_{1}}, \ldots, e^{j \phi k_{K-1}}\right),
\end{gathered}
$$

and

$$
\mathbf{A}=\left(\begin{array}{cccc}
1 & 1 & \ldots & 1 \\
k_{0} & k_{1} & \ldots & k_{K-1} \\
\ldots & \ldots & & \ldots \\
k_{0}^{N} & k_{1}^{N} & \ldots & k_{K-1}^{N}
\end{array}\right) .
$$

Finally, we can get $\overline{\mathbf{d}}_{\mathbf{i}}=(\mathbf{I}-\mathbf{P}) \mathbf{d}_{i}+\mathbf{P} \boldsymbol{\Phi}^{\mathbf{H}} \overline{\mathbf{d}}_{\mathbf{i}-1}$, where $\mathbf{P}=\boldsymbol{\Phi}^{H} \mathbf{A}^{H}\left(\mathbf{A} \mathbf{A}^{\mathbf{H}}\right)^{-} \mathbf{A} \boldsymbol{\Phi}$. The deducing detail can be referred to [1].

\section{Comparison of the OFDM/OQAM and N-continuous OFDM System}

\subsection{Comparison of the Out-of-band Radiation}

Because the pulse shape with lower side lobe such as the EGF shape is used in the OFDM/OQAM system, the final transmitting signal has very low out-of-band radiation naturally. The EGF shape is defined as [6]

$$
\begin{aligned}
z_{a, v_{0}, \tau_{0}}(t)= & \frac{1}{2}\left[\sum_{k=0}^{\infty} d_{k, a, v_{0}}\left[g_{\alpha}\left(t+\frac{k}{v_{0}}\right)+g_{\alpha}\left(t-\frac{k}{v_{0}}\right)\right]\right] \\
& \cdot \sum_{l=0}^{\infty} d_{l, 1 / \alpha, \tau_{0}} \cos \left(2 \pi l \frac{t}{\tau_{0}}\right)
\end{aligned}
$$

where $g_{\alpha}(t)$ is the Gaussian function defined by

$$
g_{\alpha}(t)=(2 \alpha)^{1 / 4} e^{-\pi \alpha t^{2}} \quad a>0 \text {. }
$$

$v_{0}$ is the frequency parameter, $\tau_{0}$ is the time parameter and $v_{0} \tau_{0}=1 / 2$. For different $a$, we can get different transmitting signal and the larger the value of $a$ is, the lower out-of-band radiation will be.

For N-continuous OFDM system, the out-of-band radiation is determined by the number of orders $N$ that having continuous derivatives. And the larger $N$ is, the lower out-of-band radiation will be.

In contrast to OFDM/OQAM, increasing of $N$ leads to not only the decrease of BER performance but also the rapid increasing of calculation complexity. Therefore, the compromise should be made between calculation consumption and system performance.

\subsection{Comparison of the BER Performance}

In OFDM/OQAM system, since the pulse shape that having immunity to the inter-symbol interference (ISI) is introduced, the CP is not necessary. Then it can provide higher bit rate or equivalently higher bit energy.

While for the N-continuous OFDM, the using of CP cannot be avoided. Furthermore, since the maximumlikelihood detector of $\mathrm{N}$-continuous is prohibitively 
complex, suboptimal iterative detector is usually used and there is a certain performance loss when the iterative time is less than 4 .

\subsection{Comparison of the Calculation Complexity}

In the OFDM/OQAM system, the complex data sources are divided into real and image part and carried out FFT calculation respectively. Then a pulse shape with the duration of $T_{c}=m T_{0}$ is met. Finally these two paths signals are added and we get the transmitting OFDM/ OQAM signal [4]. Therefore the OFDM/OQAM system calculation consumption comprises of

$$
2 M \log _{2} M+4 m M
$$

real multiplications and $2 M \log _{2} M+4(m-1) M$ real additions.

For N-continuous OFDM, the system calculation consumption comprises of $8 M^{2}$ real multiplications and $8 M^{2}-2 M$ real additions. Because both multiplications and additions calculation are directional proportional to the square of the number of subcarriers $M$. Therefore the calculation consumption increases rapidly for a large $M$.

Taking the number of subcarriers $M=256$ and the length the of pulse shape is four times of the length of OFDM symbol, i.e., $m=4$, for example, the numerical comparison of calculation complexity is listed in Table 1. It is shown that the calculation complexity of N-continuous OFDM is far more than that of the OFDM/ OQAM system.

\section{Simulation Results}

In this section, it aims to compare the out-of-band radiation and BER performance of OFDM/OQAM with the $\mathrm{N}$-continuous system. Unless other wise stated, the simulation parameters are: the number of subcarriers is $\mathrm{M}=512$, sampling time $\mathrm{Ts}=1 / 15 \mathrm{~ms}$, CP length $\mathrm{Tg}=$ 144Tsamp, the data is modulated by 16QAM. A 3GPP EVA fading channel is combined without carrier frequency offset and synchronization errors. The power spectrum is estimated by Welch's averaged periodogram method with sampling duration Tsamp, a 4096-sample Hanning window and 512-sampleoverlap. And the simulation results are shown in Figure 1 and Figure 2.

Figure 1 shows the results of BER performance of $\mathrm{N}$ continuous OFDM and OFDM/OQAM, and the results of original OFDM are also given. It can be seen that $\mathrm{N}$ continuous OFDM has poorer performance than original

Table 1. Numerical comparison of calculation complexity.

\begin{tabular}{ccc}
\hline scheme & real multiplication & addition real \\
\hline OFDM/OQAM & 8192 & 7168 \\
N-continuou & 524288 & 523776 \\
OFDM & & \\
\hline
\end{tabular}

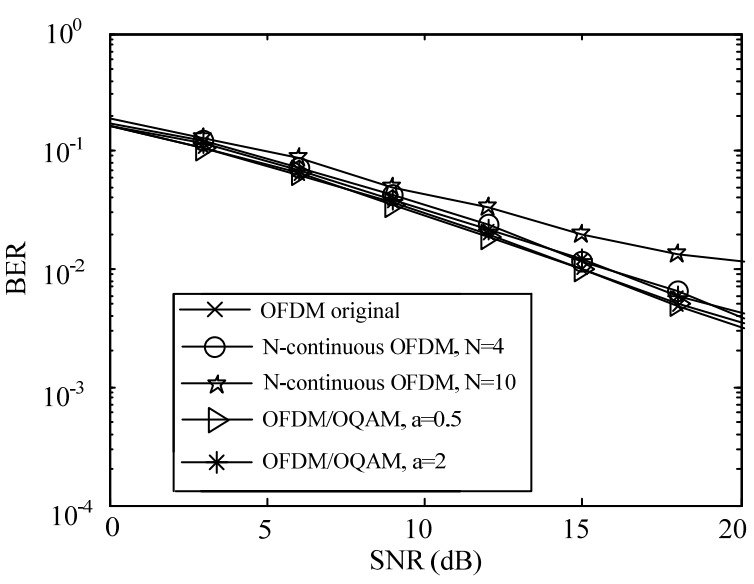

Figure 1. Comparison of BER performance of $\mathrm{N}$-continuous OFDM and OFDM/OQAM.

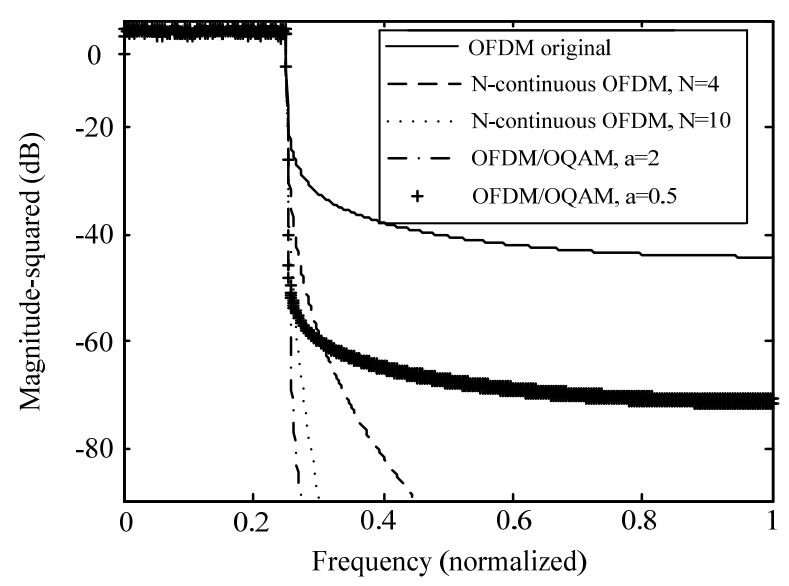

Figure 2. Comparison of out-of-band radiation of N-continuous OFDM and OFDM/OQAM.

OFDM and the situation is more serious when the $\mathrm{N}$ is up to 10 . On the other hand, for OFDM/OQAM system, it has similar performance with origin OFDM, and the performance loss is negligible even for $\alpha=2$.

In Figure 2, it shows the power spectral density of Ncontinuous OFDM and OFDM/OQAM, together with the results of original OFDM. The results imply that the $\mathrm{N}$ continuous OFDM has lower out-of-band radiation than original OFDM. And the larger $\mathrm{N}$ is, the lower out-ofband radiation will be. While for OFDM/OQAM system, it has a sharp decay at the edge of the main lobe, which is better for transmission in means of avoiding adjacent interference. And the parameter $\alpha$ has a certain effect on the out-of-band radiation. When $\mathrm{N}=10, \mathrm{~N}$-continuous OFDM is a little better than that of OFDM/OQAM with $\alpha=0.5$. But at same time, the calculation consumption is very large.

\section{Conclusions}

In this paper, the performance of OFDM/OQAM and 
$\mathrm{N}$-continuous OFDM are compared with respect to outof-band radiation, BER performance and calculation complexity with different system parameters. We show that $\mathrm{N}$-continuous OFDM is only designed to suppress the out-of-band radiation, but this benefit is acquired with large calculation consumption increase. The advantages of OFDM/OQAM lie in that it has not only the nature advantage of low out-of-band radiation, but also the strong immunity to ISI and inter-carrier interference simultaneously. When the length of pulse shape is suitable selected, while with moderate complexity increase. In whole, OFDM/OQAM system outperforms the N-continuous OFDM system.

\section{Acknowledgements}

This work is supported in part by the National Science Foundation of China under Grant number 61101101, National Grand Special Science and Technology Project of China under Grant No. 2010ZX03006-002-02,, Program for New Century Excellent Talents in University of China ((NCET110058), the Foundation Project of National Key Laboratory of Science and Technology on Communications under Grant 9140C020404120C0201, and Key Laboratory of Universal Wireless Communications, Beijing university of Posts and Telecommunications, Ministry of Education, P.R.China (No. KFKT-
2012102).

\section{REFERENCES}

[1] J. van de Beek and F. Berggren, "N-continuous OFDM," IEEE Communications Letters, Vol. 13, No. 1, 2009, pp. 1-3. doi:10.1109/LCOMM.2009.081446

[2] B. Le Floch, M. Alard and C. Berrou, "Coded Orthogonal Frequency Division Multiplex,” Proceedings of IEEE, Vol. 83, 1995, pp. 982-996. doi:10.1109/5.387096

[3] P. Siohan and C. Roche. "Cosine-Modulated Filterbanks Based on Extended Gaussian Function," IEEE Transactions on Signal Processing, Vol. 48, No. 11, 2000, pp. 3052-3061. doi:10.1109/78.875463

[4] J. F. Du, S. Signell, "Time Frequency Localization of Pulse Shaping Filters in OFD/OQAM Systems,” 6th International Conference on Information, Communications \& Signal Processing, Dec. 2007.

[5] J. van de Beek and F. Berggren, "Out-of-band Power Suppression in OFDM,” IEEE Communications Letters, Vol. 12, No. 9, 2008, pp. 609-611. doi:10.1109/LCOMM.2008.080587

[6] C. Roche and P. Siohan, "A Family of Extended Gaussian Functions with An Early Optimal Localization Property," First International Workshop Multi-Carrier Spread-Spectrum, 1997, pp. 179-186. doi:10.1007/978-1-4615-6231-3_21 